Бердимуратов А.М. Разрешимость задачи Коши-Паламодова в классе обобщенных функций бесконечного порядка

УДК 517.9

DOI: $10.21779 / 2542-0321-2021-36-4-61-67$

\title{
А.М. Бердимуратов
}

\section{Разрешимость задачи Коши-Паламодова в классе обобщенных функций бесконечного порядка}

Лисьвенский филиал Пермского национального исследовательского политехнического университета; Россия, 618902, Лысьва, ул. Ленина, 2; aman2460@таil.ru

В статье рассматривается проблема продолжения обобщенных решений однородных систем дифференциальных уравнений в частных производных с постоянными коэффициентами.

Проблемой продолжения решений таких систем занимались Л. Эренпрайс и Б. Мальгранж, в работах которых была установлена возможность продолжения решения из окрестности границы области вовнутрь области для переопределенных систем. Задачи такого рода были впервые сформулированы Л. Эренпрайсом, а более сильные результаты получены Б. Мальгранжом, В.П. Паламодовым и В.В. Грушиным как следствие теорем о разрешимости общих систем.

В.П. Паламодов сформулировал более точные теоремы о возможности продолжения обобщенных решений, заданных в окрестности границы области в наиболее важных ситуациях и при более слабом условии. То есть условия типа слабой гиперболичности на оператор с постоянными коэффициентами, накладываемыми на бесконечно удаленную часть характеристического множества оператора с постоянными коэффициентами задачи Коши-Паламодова. Работа посвящена нахождению решения в классе обобщенных функций бесконечного порядка.

Ключевые слова: слабо гиперболический оператор, конус выпукльй, диск выпукльий, гиперплоскость, алгебраическое многообразие, характеристическое множество, компакт, пространства Жеврея, несобственная точка, норма конечная.

\section{Введение}

Рассмотрим Жевреевские пространства финитных функций и сопряженные пространства. Для любого $B>0$ и $\beta>1$ через $\mathrm{D}_{F}^{\beta, B}$ обозначим пространство бесконечно дифференцируемых функций в $R^{n}$ с носителями, принадлежащими компакту $\mathrm{F}$ и конечной нормой вида.

$$
\|\varphi\|^{\beta, B}=\sup _{j} \frac{\max \left|D^{j} \varphi(\xi)\right|}{B^{|j|}|j|^{j j \beta}}
$$

Пусть $\left(\mathrm{D}_{F}^{\beta, B}\right)^{\prime}-$ сопряженное пространство: норму в этом пространстве мы будем обозначать через $\|\cdot\|_{F}^{\beta, B}$.

Рассмотрим класс основных функций $\mathrm{D}_{F}^{\beta}=\bigcap_{B>0} \mathrm{D}_{F}^{\beta, B}$.

Введем в нем счетное число норм $\|\cdot\|^{\beta, B}, B=\frac{1}{k}, k=1,2, \ldots \ldots$ 
Бердимуратов А.М. Разрешимость задачи Коши-Паламодова в классе обобщенных функций бесконечного порядка

Обозначим через $\mathrm{U}_{F}^{\beta}$ пространство линейных непрерывных функционалов над пространством $\mathrm{D}_{F}^{\beta}$.

По свойству линейного непрерывного функционала каждый элемент $\mathrm{U}_{F}^{\beta}$ непрерывен по некоторой форме $\|\cdot\|^{\beta, B}$.

Рассмотрим произвольную однородную систему линейных дифференциальных уравнений с постоянными коэффициентами:

$$
\begin{aligned}
& P_{11}(D) u_{1}+\ldots+P_{1 s}(D) u_{s}=0 \\
& P_{t 1}(D) u_{1}+\ldots+P_{t s}(D) u_{s}=0
\end{aligned}
$$

где $\mathrm{P}_{\mathrm{ij}}(\mathrm{D}) i=\overline{1, t} \quad j=\overline{1, s}$ - произвольные линейные дифференциальные операторы с постоянными коэффициентами, а числа $t$ и $s$ произвольны.

Такую систему мы будем записывать в матричной форме:

$$
P(D) u=0
$$

где $u=u_{1}, \ldots, u_{s}-$ неизвестная вектор-функция.

Пусть $F$ компакт в $R^{n}$. Пространство непрерывных линейных функционалов над пространством $\mathrm{D}_{F}^{\beta}$ обозначим через $\mathrm{U}_{F}^{\beta}$.

Пусть $N$-характеристическое множество оператора $P^{(D)}$ в $C^{n}$, а тело $N^{\prime} \subset C^{n}$ образовано бесконечно много комплексными прямыми, отвечающими несобственным точкам алгебраического многообразия $N$. Через $v^{\prime}$ обозначим множество точек в $\mathrm{R}_{y}^{n}$, которые являются мнимыми частями точек множества $N^{\prime}$.

Определение 1. В [1] характеристическим множеством системы (1) и оператора $P(D)$ называется алгебраическое многообразие $N=\left\{z \in C^{n} ; \operatorname{rang} p(z)<s\right\}$, где $p(z)$ матрица, полученная заменой операторов $P_{i j}(D)$ многочленами $p_{i j}(z)$.

Пусть $N$ - некоторое алгебраическое многообразие в $C^{n}$. Пространство $C^{n}$ вложим в $C^{n+1}$ с помощью отображения $z \rightarrow(1, z)$. Пусть $H(N)$ - совокупность всех однородных многочленов в $C^{n+1}$, отображающихся в нуль на $N$.

Определение 2. Любая точка вида $(0, \mathrm{z}), z \in C^{n}$, в которой обращаются в нуль все многочлены из $\mathrm{H}(N)$, называется несобственной точкой многообразия $N$. Заметим, что если размерность рассматриваемого диска $G(r)$ равна $n-1$, то имеется ровно два конуса $\Gamma^{\delta}(r)$ размерности $n$, которые опираются на этот диск. Например, если диск лежит в плоскости $z=0$, то в вершине одного из этих конусов $z=\delta \cdot r$, а в вершине второго конуса $z=-\delta \cdot r$.

Если же размерность $d$ диска $G(r)$ меньше $n-1$, то имеется бесконечно много

62

Вестник Дагестанского государственного университета.

Серия 1. Естественные науки. 2021. Том 36. Вып. 4 
конусов размерности $d+1$ с фиксированным угловым параметром $\delta$, которые опираются на этот диск.

Их объединение $\Delta^{\delta}(r)$ есть выпуклое $n$-мерное тело, имеющее вращательную симметрию вокруг подпространства, в котором лежит диск $G(r)$. Можно рассматривать задачу о продолжении решений из окрестности этого диска в окрестность тела $\Delta^{\delta}(r)$. Такие задачи мы будем называть аналогами классических задач КошиПаламодова в классах обобщенных функций бесконечного порядка $\mathrm{U}_{F}^{\beta}$. Пусть $y$ - вектор в $\mathrm{R}^{n}$, через $H_{y}$ обозначим гиперплоскость в $\mathrm{R}^{n}$, ортогональную к вектору $y$. Через $G(r)$ обозначим $(n-2)$-мерный диск, лежащий в $H_{y}$ с центром в начале координат, радиуса $r>0$. Через $\Delta^{\delta}(r)$, где $\delta>0$, обозначим объединение бес жества прямых конусов с фиксированным угловым параметром $\delta$ и общим основанием $G(r)$, которое опирается на этот диск, и высотами, равными $\delta \cdot r$.

Oпределение 3. Оператор $P(D)$ будем называть однородным, если матрицы $p$ в системе (1) образованы однородными полиномами одинакового порядка.

Пусть $N$ - некоторое алгебраическое многообразие в $C^{n}$. Пусть $\mathrm{H}(N)$ - совокупность всех однородных многочленов в $C^{n+1}$, отображающихся в нуль на $N$.

\section{Постановка задачи}

При каких условиях всякое обобщенное решение системы (1), определенное в окрестности диска $G(r)$, может быть продолжено в окрестность тела $\Delta^{\delta}(r)$ ? Эту задачу можно рассматривать как некоторый аналог классической задачи Коши-Паламодова для обобщенных решений: вместо значений решения и его производных решение задается сразу в некоторой окрестности [1, гл. VI, § 4, теор. 1, сл. 1].

Обозначим через $N$ характеристическое множество системы (1) в комплексном пространстве $C^{n}$. Обозначим через $v$ проекцию множества $N$ на мнимое подпространство в $C^{n}$, обозначаемое через $R_{y}^{n}$. Если оператор $P(D)$ однородный, то многообразие $N$ является телом, а отсюда следует, что множество $v$ тоже является телом.

Сформулируем теорему Паламодова, полученную в [1, гл. IV, § 4, теор. 2].

Теорема 1 [1]. Если $L$ и $T \mathrm{C} L-$ выпуклые компакты в $\mathrm{R}^{n}$, то для любого $\beta>1$ и В $>0$ любая функция $u \in\left[\left(\mathrm{D}_{T}^{\beta, B}\right)^{\prime}\right]^{s}$, являющаяся обобщенным решением системы (1) в классе $\mathrm{U}_{T}^{\beta}$, может быть записана в виде

$$
(\overline{u, \varphi})=\sum_{\lambda=0}^{\iota} d^{\lambda}\left(z, D_{z}\right) \emptyset^{*} \mu^{\lambda}
$$

для любой функции $\varphi \in\left[\mathrm{D}_{L}^{\beta, B}\right]^{s}$, причем векторы $\mu^{\lambda}$ таковы, что 


$$
\sum_{\lambda=0}^{l} \int_{N^{\lambda}}\left(\frac{\beta}{e}\left(\frac{|z|}{D}\right)^{\frac{1}{\beta}}\right){ }_{L}(y)\left|\mu^{\lambda}\right| \leq c\|u\|_{T}^{\beta, B},
$$

где $\|u\|_{T}^{\beta, B}$ есть норма $u$ как элемент пространства $\left[\left(\mathrm{D}_{T}^{\beta, B}\right)^{\prime}\right]^{s}$, а число $D>0$ и зависит только от $\beta$ и $B$.

Обратно: всякий функционал $u$, определенный формулой (3), с конечной величиной

$$
\sum_{\lambda=0}^{l} \int_{N^{\lambda}}\left(-\frac{\beta}{e}\left(\frac{|z|}{D^{\prime}}\right)^{\frac{1}{\beta}}\right) \mathrm{J}_{L}(y)\left|\mu^{\lambda}\right|<\infty
$$

при некотором положительном $D^{\prime}$ принадлежит пространству $\left[\left(\mathrm{D}_{L}^{\beta, B^{\prime}}\right)^{\prime}\right]^{s}$ и является обобщенным решением системы (1) в классе $\mathrm{U}_{L}^{\beta}$.

Приведем аналог вышеприведенной теоремы 1, которая дает условия продолжаемости решений системы (1) в классе обобщенных функций бесконечного порядка.

Теорема 2. Пусть $P(D)$ - слабо гиперболический оператор порядка $m$, а $v^{\prime} \neq R_{y}^{n}$ и $y_{0} \in R_{y}^{n} \backslash v^{\prime}$, число $r>0$ произвольное.

Тогда существуют число $\delta>0$ и число $h<1$, зависящее от оператора $p(D)$, такое, что для любого $\beta$, удовлетворяющего условию $\beta>1$ для любой окрестности $L$ диска $G(r)$, лежащего в гиперплоскости $H_{y_{0}}$, существует окрестность $T$ тела $\Delta^{\delta}(r)$ такая, что всякое решение $u$ системы (1) из класса $\left[\mathrm{U}_{L}^{\beta}\right]^{s}$ может быть продолжено обобщенной функцией $v$ как решение системы (1) в классе $\left[\mathrm{U}_{T}^{\beta}\right]^{s}$.

Доказательство теоремы 2. Пусть $\left\{\varepsilon_{\alpha}\right\}$ - строго возрастающая последовательность положительных чисел, $-\infty<\alpha<\infty$ такая, что $\left\{\varepsilon_{\alpha}\right\} \rightarrow 0$ при $\alpha \rightarrow-\infty$, и обозначим через $G_{\alpha}$ замкнутую $\varepsilon_{\alpha}$-окрестность диска $G_{\propto}$. Пусть $u$ - произвольное обобщенное решение системы (1), определенное в окрестности $G_{\alpha}$, тогда в силу того, что $\left\{\varepsilon_{\alpha}\right\} \rightarrow 0$ при $\alpha \rightarrow-\infty$, найдется такое $\propto$, что обобщенная функция $u$ будет определена и являться решением системы (1) на компакте $G_{\alpha}$.

Применяя к компактам $G_{\alpha}$ и $G_{\alpha-2}$ и функционалу и вышеприведенную теор. 1 , получим, что обобщенная функция $u \in\left[\mathrm{U}_{G_{\alpha}}^{\beta}\right]^{s}$, являющаяся решением системы (1), в классе $\left[\mathrm{U}_{G_{\alpha}}^{\beta}\right]^{s}$ для любой функции $\varphi \in\left[\mathrm{D}_{G_{\alpha-2}}^{\beta}\right]^{s}$ допускает представление в виде

$$
(\overline{u, \varphi})=\sum_{\lambda=0}^{\ell} d^{\lambda}\left(z, D_{z}\right) \varphi^{*} \mu^{\lambda},
$$

в котором меры $\mu^{\lambda}$ сосредоточены на множествах $N^{\lambda}$ и таковы, что при некотором 64 Вестник Дагестанского государственного университета. Серия 1. Естественные науки. 2021. Том 36. Вып. 4 
$\beta>0$

$$
\sum_{\lambda=0}^{l} \int_{N^{\lambda}} \exp \left(-\left(\frac{|z|}{B}\right)^{\frac{1}{\beta}}\right) \mathbf{J}_{G_{\alpha-2}}(y)\left|\mu^{\lambda}\right|<\infty .
$$

Допустим, мы доказали, что при некотором $\gamma$ и каком-то $B^{\prime}>0$

$$
\sum_{\lambda=0}^{l} \int_{N^{\lambda}} \exp \left(-\left(\frac{|z|}{B^{\prime}}\right)^{\frac{1}{\beta}}\right) \mathrm{J}_{\Delta_{\delta}}(y)\left|\mu^{\lambda}\right|<\infty .
$$

Тогда в силу второй части формулы теоремы $1(\overline{u, \varphi})=\sum_{\lambda=0}^{\ell} d^{\lambda}\left(z, D_{z}\right) \varphi^{*} \mu^{\lambda}$, для любой функции $\varphi \in\left[\mathrm{D}_{G_{\alpha-2}}^{\beta}\right]^{s}$, т. е. $v \in\left[\mathrm{U}_{\Delta_{v-2}}^{\beta}\right]^{s}$. Это решение $\vartheta$ очевидно совпадает с решением $u$ на классе $\left[\mathrm{D}_{G_{\alpha-2} \cap \Delta_{y-2}}^{\beta}\right]^{s}$, где $G_{\alpha-2} \cap \Delta_{v-2}$ является окрестностью диска $G(r)$.

Для доказательства теоремы 2 нужно доказать неравенство

$$
\sum_{\lambda=0}^{\ell} \int_{N^{\lambda}} \exp \left(-\left(\frac{|z|}{B^{\prime}}\right)^{\frac{1}{\beta}}\right) \mathrm{J}_{\Delta_{\delta}}(y)\left|\mu^{\lambda}\right|<\infty .
$$

Так как

$$
\begin{aligned}
& \sum_{\lambda=0}^{l} \int_{N^{\lambda}} \exp \left(-\left(\frac{|z|)^{\frac{1}{\beta}}}{B^{\prime}}\right)^{\frac{1}{\beta}} \mathrm{J}_{\Delta_{v}}(y)\left|\mu^{\lambda}\right| \leq\right. \\
& \sup _{z \in N} \frac{\mathrm{J}_{\Delta_{v}}(y) \exp \left(-B_{0}|z| \frac{1}{\beta}\right)}{\mathrm{J}_{\mathrm{G}_{\alpha-2}}(y)} \sum_{\lambda=0}^{l} \int_{N^{\lambda}} \exp \left(-\left(\frac{|z|}{B}\right)^{\frac{1}{\beta}}\right) \mathrm{J}_{\mathrm{G}_{\alpha-2}}(y)\left|\mu^{\lambda}\right|,
\end{aligned}
$$

достаточно показать, что первый сомножитель этого неравенства конечен:

$$
\begin{aligned}
& \ln \sup _{z \in N} \frac{\mathrm{J}_{\Delta_{v}}(y) \exp \left(-B_{0}|z|^{\frac{1}{\beta}}\right)}{\mathrm{J}_{\mathrm{G}_{\alpha-2}}(y)}= \\
& \sup _{z \in N}\left[\sup _{\xi \in \Delta_{v}}(y, \xi)-\sup _{\xi \in \mathrm{G}_{v-2}}(y, \xi)-B_{0}|z|^{\frac{1}{\beta}}\right]<\infty .
\end{aligned}
$$

Теорема доказана.

\section{Заключение}

Получено условие на несобственную часть характеристического множества системы (1), обеспечивающее продолжаемость обобщенных решений бесконечного порядка с окрестности диска в окрестность n-мерного тела.

\section{Литература}


Бердимуратов А.М. Разрешимость задачи Коши-Паламодова в классе обобщенных функций бесконечного порядка

1. Паламодов В.П. Линейные дифференциальные операторы с постоянными коэффициентами. - М.: Наука, 1967. - 488 с.

2. Паламодов В.П. Полиномиальные идеалы и уравнения в частных производных // УМН. - 1963. - Т. 18, № 2 (110). - С. 164-168.

3. Паламодов В.П. Общий вид решений линейных дифференциальных уравнений с постоянными коэффициентами // ДАН СССР. - 1962. - Т. 143, № 6. C. $1278-1281$.

4. Бердимуратов А.М. Теория разрешимости задачи Коши-Паламодова в пространствах обобщенных функций//Тезисы докл. Межд. апрельской мат. конф., посвященной 75-летию акад. НАН РК Кальменова Т.Ш., (г. Алматы, 5-8 апреля 2021 г.).

5. Бердимуратов А.M. О единственности обобщенных решений систем дифференциальных уравнений с постоянными коэффициентами // Вестник Бурятского государственного университета. Математика, информатика. - 2021. - № 1. - С. 24-33.

6. Бердимуратов А.M. Метод экспоненциального представления Паламодова и его приложения к некоторым аналогам классических задач в пространствах обобщенных функций. - Бишкек, 2017. - 134 с.

7. Бердимуратов А.М. Метод экспоненциального представления в пространствах обобщенных функций. - LAP Lambert Academic Publishig, - 2017. - 96 c.

8. Грушин B.B. О решениях с изолированными особенностями для уравнений в частных производных с постоянными коэффициентами // ТММО. - 1966. - Т. 15. C. 262-278.

9. Ehrenpris L. A new proof and an extension of Hartogs theorem // Bull. Amer. Soc. - 1961. - Vol. 67, № 5. - C. 507-509.

10. Malgrang B. Systemes differentiels a coefficients constants // Seminaire Bourbaki. - Paris, 1962. - Vol. 63. - № 246.

\section{References}

1. Palamodov V.P. Linear differential operators with constant coefficients. - Moscow, Nauka, 1967. $488 \mathrm{c.}$

2. Palamodov V.P. of Polynomial ideals and partial differential equations, Russian math 18, 2 (110), P. 164-168 (1963).

3. Palamodov V.P. General form of the solutions of linear differential equations with constant coefficients, DAN SSSR 143, No. 6, 1278-1281(1962).

4. Berdimuratov A.M. The theory of solvability of the Koshi-Palmodov problem in spaces of generalized functions // abstracts of the international April Mathematical conference dedicated to the 75th anniversary of Academician of the NAS RK Kalmenov T.Sh., 5-8 April 2021, page 20. g. Almaty.

5. Berdimuratov A.M. On the uniqueness of generalized solutions of systems of differential equations with constant coefficients // Bulletin of the Buryat State University. Mathematics, Computer Science", № 1, 2021. P. 24-33.

6. Berdimuratov A.M. The Palamodov exponential representation method and its applications to some analogues of classical problems in spaces of generalized functions. Bishkek, $2017.134 \mathrm{c}$.

7. Berdimuratov A.M. Method of exponential representation in spaces of generalized functions. - LAP Lambert Academic Publishig, 2017. - 96 p.

8. Grushin V.V. On solutions with isolated singularities for partial differential equations with constant coefficients. TMMO 15, 262-278 (1966).

9. Ehrenpris L. A new proof and an extension of Hartogs theorem, Bull. Amer. Soc.

66

Вестник Дагестанского государственного университета.

Серия 1. Естественные науки. 2021. Том 36. Вып. 4 
Бердимуратов А.М. Разрешимость задачи Коши-Паламодова в классе обобщенных функций бесконечного порядка

67, № 5 1961, 507-509.

10. Malgrang B. Systemes differentiels a coefficients constants, Seminaire Bourbaki, Paris, 1962/63, № 246.

Поступила в редакиию 20 октября 20212.

UDC 517.9

DOI: $10.21779 / 2542-0321-2021-36-4-61-67$

\section{Solvability of Cauchy-Palamodov Problem in the Class of Generalized Functions of Infinite Order}

\section{A.M. Berdimuratov}

Lysva branch of the Perm National Research Polytechnic University; Russia, 618902, Lysva, Lenin st., 2; aman2460@mail.ru

The paper develops the problem of generalized solutions of homogeneous systems of differential equations in partial derivatives with constant coefficients.

The given problem was dealt with by L. Ehrenpreis and B. Malgrange. In their works the possibility of continuation of solutions from the vicinity of the boundary of the region into the interior of the domain for redefined systems was established. This idea was first formulated by L. Erenpreis and stronger results were obtained by B. Malgrange, V.P. Palamodov and V.V. Gruzinim as a consequence of theorems on time determination of the overall systems.

V.P. Palamodov established more precise theorems on the possibility of generalized solutions continuation given in the neighborhood of the area boundary in the most important situations and under a weaker condition - to be exact - the conditions of weak hyperbolicity on the abstract function with fixed factors applied to the infinitely distant part of the operator characteristic set with constant coefficients of Cauchy-Palamodov equation. The research helps find solution in the class of generalized functions of infinite order.

Keywords: weakly hyperbolic operator, convex cone, convex disc, hyperplane, algebraic variety, characteristic set, compact set, Gevrey spaces, improper point, finite norm.

Received 20 October 2021 